\title{
A GENERALIZATION OF THE ERLANG FORMULA OF TRAFFIC ENGINEERING
}

\author{
V. ANANTHARAM * \\ Department of Electrical Engineering, Phillips Hall, Cornell University, Ithaca, New York 14853, \\ U.S.A.
}

\section{B. GOPINATH and D. HAJELA}

Bell Communications Research, Inc., 435 South Street, Morristown, New Jersey 07960, U.S.A.

Received 9 September 1986

Revised 16 November 1987

\begin{abstract}
Calls arrive at a switch, where they are assigned to any one of the available idle outgoing links. A call is blocked if all the links are busy. A call assigned to an idle link may be immediately lost with a probability which depends on the link. For exponential holding times and an arbitrary arrival process we show that the conditional distribution of the time to reach the blocked state from any state, given the sequence of arrivals, is independent of the policy used to route the calls. Thus the law of overflow traffic is independent of the assignment policy. An explicit formula for the stationary probability that an arriving call sees the node blocked is given for Poisson arrivals. We also give a simple asymptotic formula in this case.
\end{abstract}

Keywords: Erlang formula, blocking probability, queueing

\section{Introduction}

Telephone calls arrive at a switching centre, which has $n$ outgoing links, grouped into $k$ trunks of $n_{1}, \ldots, n_{k}$ links respectively. A call assigned to a link in trunk $\alpha$ is immediately lost with probability $\left(1-\epsilon_{\alpha}\right)$ where the different loss probabilities $\left(1-\epsilon_{\alpha}\right)$ model the cumulative effects of the topology of the network and the congestion of other links and nodes downstream of the node under consideration. If the call is successful, the link immediately becomes busy for a holding or conversation time, which is independent of the arrival process and exponentially distributed, with mean $1 / \mu$. The number of idle links in each trunk, $0 \leqslant i_{1} \leqslant n_{1}, \ldots, 0 \leqslant i_{k} \leqslant n_{k}$, specifies the state, $\left(i_{1}, \ldots, i_{k}\right)$, of the node. For a stage $I=\left(i_{1}, \ldots, i_{k}\right)$ and $1 \leqslant \alpha \leqslant k$ such that $i_{\alpha} \neq 0$, let $I_{\alpha}$ denote the state

$$
I_{\alpha}=\left(i_{1}, \ldots, i_{\alpha-1}, i_{\alpha}-1, i_{\alpha+1}, \ldots, i_{k}\right) \text {. }
$$

* Work on this paper was done while the author was at Bellcore and at Berkeley. 
The policy for routing incoming calls is specified by numbers $U\left(I, I_{\alpha}\right)$ satisfying

$$
\begin{aligned}
& U\left(I, I_{\alpha}\right) \geqslant 0, \\
& \sum_{1 \leqslant \alpha \leqslant k, i_{\alpha} \neq 0} U\left(I, I_{\alpha}\right)=1 \text { if } I \neq(0, \ldots, 0),
\end{aligned}
$$

so that, when the node is in state $I$, and the policy is $U$, for $1 \leqslant \alpha \leqslant k$ with $i_{\alpha} \neq 0$ an incoming call is assigned to trunk $\alpha$ with probability $U\left(I, I_{\alpha}\right)$. When all outgoing links are busy the switch is said to be in the blocked state.

In general this models the allocation of resources of different types where a resource allocated to a request has a certain probability of relinquishing it immediately or servicing it from an exponentially distributed interval. When the $\epsilon_{\alpha}=0$ we get a model that has been extensively studied in the literature (Benes [1], Khintchine [3], Takacs [5] and the references therein). For Poisson arrivals, the well known expression for the stationary probability that the switch is in the blocked state is called the Erlang formula. The refined model of the first paragraph was proosed by Gopinath, Garcia and Varaiya, and we refer the reader to Gopinath et al. [2] for further motivation for its introduction. Poisson arrivals are considered in Gopinath et al. [2], and the remarkable fact is proved that the distribution of the time to reach the blocked state from any initial state is independent of the assignment policy. A formula is conjectured for the blocking probability when there are two types of outgoing trunks.

In sections 2 and 3 we establish the conjectured formula of Gopinath et al. [2] for an arbitrary number of outgoing links, thus generalizing the Erlang formula. In section 4 we show that, in fact, for an arbitrary arrival process, the conditional distribution of the time to reach the blocked state starting from any initial state, given the sequence of arrivals, is independent of the routing policy, so that the law of overflow traffic is independent of policy. In section 5 we give a simple asymptotic formula for the blocking probability formula derived in section 3 .

\section{Poisson arrivals; the case $k=2$}

The evolution of the state of the node is described by a continuous time finite state Markov process with state space

$$
X=\left\{I=\left(i_{1}, \ldots, i_{k}\right) \text { such that } 0 \leqslant i_{1} \leqslant n_{1}, \ldots, 0 \leqslant i_{k} \leqslant n_{k}\right\} .
$$

Recall that, given state $I$ and $1 \leqslant \alpha \leqslant k$ such that $i_{\alpha} \neq 0$, we let

$$
I_{\alpha}=\left(i_{1}, \ldots, i_{\alpha-1}, i_{\alpha}-1, i_{\alpha+1}, \ldots, i_{k}\right) .
$$

Similarly, given $1 \leqslant \beta \leqslant k$ such that $i_{\beta} \neq n_{\beta}$, let

$$
I_{\beta}=\left(i_{1}, \ldots, i_{\beta-1}, i_{\beta}+1, i_{\beta+1}, \ldots, i_{k}\right) \text {. }
$$


$\left(0, n_{2}\right)$

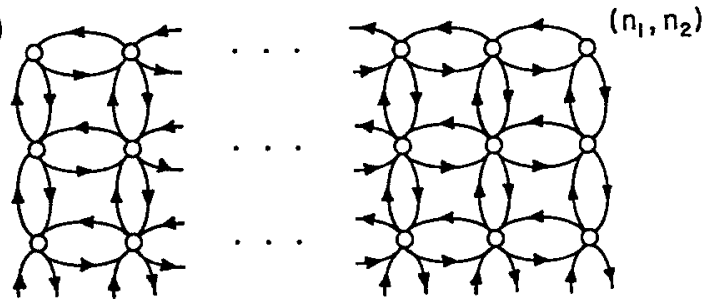

$(0,0)$

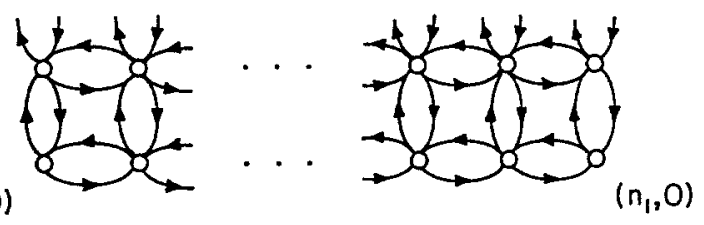

Fig. 1.

If the policy is $U$, the transition rate matrix of the Markov process is given by

$$
\begin{aligned}
& R_{U}(I, J)=0 \quad \text { if } J \neq I_{\alpha}, J \neq I^{\beta}, J \neq I, \\
& R_{U}\left(I, I_{\alpha}\right)=\lambda_{\alpha} U\left(I, I_{\alpha}\right) \text { where } \lambda_{\alpha}=\epsilon_{\alpha} \cdot \lambda, \\
& R_{U}\left(I, I^{\beta}\right)=\left(n_{\beta}-i_{\beta}\right) \mu, \\
& R_{U}(I, I)=-\sum_{1 \leqslant \alpha \leqslant k, i_{\alpha} \neq 0} \lambda_{\alpha} U\left(I, I_{\alpha}\right)-\sum_{1 \leqslant \beta \leqslant k, i_{\beta} \neq n_{\beta}}\left(n_{\beta}-i_{\beta}\right) \mu .
\end{aligned}
$$

Let $\left\{p_{U}(I), I \in X\right\}$ be the steady state distribution of this Markov process. The blocking probability $p_{b}$ is $p_{U}[(0, \ldots, 0)]$, and, by Gopinath et al. [2], we know that $p_{b}$ is independent of the routing policy. Our method will be to calculate the formula for $p_{b}$ by calculating the steady state distribution for a special analytically convenient policy, which has the property that the transition rate matrix of the associated Markov process is "decoupled" in a way that will become clear below.

In this section we consider the case $k=2$, in which our idea for calculating the Erlang formula for the blocking probability is most easily understood. The transition matrix is conveniently thought of by means of the state diagram of fig. 1. Left to right and down to up transitions represent links becoming free, while transitions in the opposite directions occur when a new call arrives. The rates associated with the arrows in the state diagram can be inferred from the equations (2.1)-(2.4). Note that they depend on the policy $U$.

A probability distribution $p(I)$ on the state space is the stationary distribution $p_{U}(I)$ iff it satisfies the balance equations

$$
\sum_{I} p(I) R_{U}(I, J)=0 \text { for each } J .
$$


These translate, in the state diagram, into one balance equation per node, the balance being the equality of the total incoming rate to the total outgoing rate. In general, for a policy $U$, the individual state to state transition rates need not be balanced. Our key observation is that there is a special policy, which is in fact unique, where these extra balance equations are satisfied by the equilibrium policy. As one would expect, this makes the calculation of the closed form generalized Erlang formula feasible. This is expressed in proposition 1, which is proved in section 3 as a special case of proposition 2 .

\section{PROPOSITION 1}

Consider the special policy for which

$$
U\left(I, I_{\alpha}\right)=\frac{i_{\alpha}}{i_{1}+i_{2}}
$$

and denote

$$
p\left(i_{1}, i_{2}\right)=\frac{\left(\begin{array}{c}
n_{1} \\
i_{1}
\end{array}\right)\left(\begin{array}{c}
n_{2} \\
i_{2}
\end{array}\right)\left(\frac{\mu}{\lambda_{1}}\right)^{i_{1}}\left(\frac{\mu}{\lambda_{2}}\right)^{i_{2}}\left(i_{1}+i_{2}\right) !}{\sum_{j_{1}=0}^{n_{1}} \sum_{j_{2}=0}^{n_{2}}\left(\begin{array}{c}
n_{1} \\
j_{1}
\end{array}\right)\left(\begin{array}{c}
n_{2} \\
j_{2}
\end{array}\right)\left(\frac{\mu}{\lambda_{1}}\right)^{j_{1}}\left(\frac{\mu}{\lambda_{2}}\right)^{j_{2}}\left(j_{1}+j_{2}\right) !} .
$$

Then we have

$$
p(I) U\left(I, I_{\alpha}\right) \lambda_{\alpha}=p\left(I_{\alpha}\right)\left(n_{\alpha}-\left(i_{\alpha}-1\right)\right) \mu
$$

for each $I$, and for each $1 \leqslant \alpha \leqslant 2$ such that $i_{\alpha} \neq 0 . p(I)$ is the steady state distribution for the policy (2.6) and the blocking probability

$$
p_{b}=\frac{1}{\sum_{j_{1}=0}^{n_{1}} \sum_{j_{2}=0}^{n_{2}}\left(\begin{array}{c}
n_{1} \\
j_{1}
\end{array}\right)\left(\begin{array}{c}
n_{2} \\
j_{2}
\end{array}\right)\left(\frac{\mu}{\lambda_{1}}\right)^{j_{1}}\left(\frac{\mu}{\lambda_{2}}\right)^{j_{2}}\left(j_{1}+j_{2}\right) !}
$$

is, in fact, the blocking probability for every routing policy.

\section{Poisson arrivals; the general case}

\section{PROPOSITION 2}

Consider the special policy for which

$$
U\left(I, I_{\alpha}\right)=\frac{i_{\alpha}}{i_{1}+\ldots+i_{k}}
$$


and denote

$$
p(I)=\frac{\left(\begin{array}{c}
n_{1} \\
i_{1}
\end{array}\right) \ldots\left(\begin{array}{c}
n_{k} \\
i_{k}
\end{array}\right)\left(\frac{\mu}{\lambda_{1}}\right)^{i_{1}} \ldots\left(\frac{\mu}{\lambda_{k}}\right)^{i_{k}}\left(i_{1}+\ldots+i_{k}\right) !}{\sum_{j_{1}=0}^{n_{1}} \ldots \sum_{j_{k}=0}^{n_{k}}\left(\begin{array}{c}
n_{1} \\
j_{1}
\end{array}\right) \ldots\left(\begin{array}{c}
n_{k} \\
j_{k}
\end{array}\right)\left(\frac{\mu}{\lambda_{1}}\right)^{j_{1}} \ldots\left(\frac{\mu}{\lambda_{k}}\right)^{j_{k}}\left(j_{1}+\ldots+j_{k}\right) !} .
$$

Then we have

$$
p(I) \cdot U\left(I, I_{\alpha}\right) \cdot \lambda_{\alpha}=p\left(I_{\alpha}\right) \cdot\left(n_{\alpha}-\left(i_{\alpha}-1\right)\right) \cdot \mu
$$

for each $I$, and each $1 \leqslant \alpha \leqslant k$ such that $i_{\alpha} \neq 0$, where $p(I)$ is the stationary distribution for the policy (3.1) and the blocking probability

$$
p_{b}=\frac{1}{\sum_{j_{1}=0}^{n_{1}} \ldots \sum_{j_{k}=0}^{n_{k}}\left(\begin{array}{c}
n_{1} \\
j_{1}
\end{array}\right) \ldots\left(\begin{array}{c}
n_{k} \\
j_{k}
\end{array}\right)\left(j_{1}+\cdots+j_{k}\right) !\left(\frac{\mu}{\lambda_{1}}\right)^{j_{1}} \ldots\left(\frac{\mu}{\lambda_{k}}\right)^{j_{k}}}
$$

is, in fact, the blocking probability for every routing policy.

\section{Proof}

The function (3.1) defines a policy because

$$
\sum_{1 \leqslant \alpha \leqslant k, i_{\alpha} \neq 0} U\left(I, I_{\alpha}\right)=1
$$

and (3.2) defines a probability distribution on the state space because $\Sigma_{I} p(I)=1$. Let $I$ be a state and $1 \leqslant \alpha \leqslant k$ be such that $i_{\alpha} \neq 0$. Then we compute

$$
\frac{p\left(I_{\alpha}\right)}{p(I)}=\frac{\left(\begin{array}{c}
n_{1} \\
i_{1}
\end{array}\right) \cdots\left(\begin{array}{c}
n_{\alpha} \\
i_{\alpha}-1
\end{array}\right) \cdots\left(\begin{array}{c}
n_{k} \\
i_{k}
\end{array}\right)}{\left(\begin{array}{c}
n_{1} \\
i_{1}
\end{array}\right) \ldots\left(\begin{array}{c}
n_{\alpha} \\
i_{\alpha}
\end{array}\right) \ldots\left(\begin{array}{c}
n_{k} \\
i_{k}
\end{array}\right)} \cdot \tau
$$

where

$$
\tau=\frac{\left(\frac{\mu}{\lambda_{1}}\right)^{i_{1}} \ldots\left(\frac{\mu}{\lambda_{\alpha}}\right)^{i_{\alpha}-1} \ldots\left(\frac{\mu}{\lambda_{k}}\right)^{i_{k}}\left(i_{1}+\ldots+i_{\alpha}-1+\ldots+i_{k}\right) !}{\left(\frac{\mu}{\lambda_{1}}\right)^{i_{1}} \ldots\left(\frac{\mu}{\lambda_{\alpha}}\right)^{i_{\alpha}} \ldots\left(\frac{\mu}{\lambda_{k}}\right)^{i_{k}}\left(i_{1}+\ldots+i_{\alpha}+\ldots+i_{k}\right) !} .
$$

Thus

$$
\frac{p\left(I_{\alpha}\right)}{p(I)}=\frac{i_{\alpha}}{n_{\alpha}+\left(i_{\alpha}-1\right)} \cdot \frac{\lambda_{\alpha}}{\mu} \cdot \frac{1}{\left(i_{1}+\cdots+i_{k}\right) !}=\frac{U\left(I, I_{\alpha}\right) \cdot \lambda_{\alpha}}{\left(n_{\alpha}-\left(i_{\alpha}-1\right)\right) \cdot \mu} .
$$

This verifies that the probability distribution (3.2) satisfies the equations (3.3). 
Next, we verify that $p(I)$ also satisfies the balance equations (2.5), which proves that $p(I)$ is the stationary distribution for the process with the policy (3.1). First observe that for any sate $J$ and $1 \leqslant \alpha \leqslant k$ such that $j_{\alpha} \neq 0$ we have

$$
\left(J_{\alpha}\right)^{\alpha}=J
$$

and for $1 \leqslant \beta \leqslant k$ such that $j_{\beta} \neq n_{\beta}$ we have

$$
\left(J^{\beta}\right)_{\beta}=J \text {. }
$$

The balance equation corresponding to column $J$ of the transition rate matrix $R_{U}$ reads

$$
\begin{gathered}
\sum_{1 \leqslant \alpha \leqslant k, j_{\alpha} \neq 0} p\left(J_{\alpha}\right) R_{U}\left(J_{\alpha}, J\right)+\sum_{1 \leqslant \beta \leqslant k, j_{\beta} \neq n_{\beta}} p\left(J^{\beta}\right) R_{U}\left(J^{\beta}, J\right) \\
+p(J) R_{U}(J, J)=0 .
\end{gathered}
$$

Note that

$$
R_{U}\left(J_{\alpha}, J\right)=R_{U}\left(J_{\alpha},\left(J_{\alpha}\right)^{\alpha}\right)=\left(n_{\alpha}-\left(j_{\alpha}-1\right)\right) \mu
$$

from equations (2.3) and (3.5). We also have

$$
R_{U}\left(J^{\beta}, J\right)=R_{U}\left(J^{\beta},\left(J^{\beta}\right)_{\beta}\right)=\lambda_{\beta} U\left(J^{\beta}, J\right)
$$

from equations (2.2) and (3.6).

Using (2.4) and the above, equation (3.7) may be rearranged to read

$$
\begin{aligned}
& \sum_{1 \leqslant \alpha \leqslant k, j_{\alpha} \neq 0}\left[p\left(J_{\alpha}\right)\left(n_{\alpha}-\left(j_{\alpha}-1\right)\right) \mu-p(J) \lambda_{\alpha} U\left(J, J_{\alpha}\right)\right] \\
& \quad+\sum_{1 \leqslant \beta \leqslant k, j_{\beta} \neq n_{\beta}}\left[p\left(J^{\beta}\right) \lambda_{\beta} U\left(J^{\beta}, J\right)-p(J)\left(n_{\beta}-j_{\beta}\right) \mu\right]=0,
\end{aligned}
$$

which holds because each individual term vanishes by (3.2). This verifies that $p(I)$ is the stationary distribution for the policy (3.1).

Finally, the blocking probability is just the stationary probability of the state $(0, \ldots, 0)$ giving (3.4). Equation (2.10) generalizes the Erlang's formula.

Explicitly evaluating the expression in (3.4) would require a large number of operations even in modest situations. Thus, it is useful to have an integral representation for $p_{b}$ from which good approximations to the closed form solution can be made, as will be seen in section 5 . This can be arrived at through the gamma-function identity

$$
n !=\int_{0}^{\infty} t^{n} \exp (-t) \mathrm{d} t
$$

Using this for $\left(j_{1}+\ldots+j_{k}\right)$ ! in (3.4) gives

$$
\begin{aligned}
p_{b}= & 1 /\left\{\sum_{j_{1}=0}^{n_{1}} \ldots \sum_{j_{k}=0}^{n_{k}}\left(\begin{array}{l}
n_{1} \\
j_{1}
\end{array}\right) \ldots\left(\begin{array}{c}
n_{k} \\
j_{k}
\end{array}\right)\left(\int_{0}^{\infty} t^{j_{1}+\ldots+j_{k}} \exp (-t) \mathrm{d} t\right)\right. \\
& \left.\times\left(\frac{\mu}{\lambda_{1}}\right)^{j_{1}} \ldots\left(\frac{\mu}{\lambda_{k}}\right)^{j_{k}}\right\} .
\end{aligned}
$$


Interchanging the summation and the integral,

$$
p_{b}=\frac{1}{\int_{0}^{\infty}\left(\sum_{j_{1}=0}^{n_{1}} \ldots \sum_{j_{k}=0}^{n_{k}}\left(\begin{array}{l}
n_{1} \\
j_{1}
\end{array}\right) \ldots\left(\begin{array}{c}
n_{k} \\
j_{k}
\end{array}\right)\left(\frac{t \mu}{\lambda_{1}}\right)^{j_{1}} \ldots\left(\frac{t \mu}{\lambda_{k}}\right)^{j_{k}}\right) \mathrm{d} t} .
$$

Finally, observing that

$$
\left(1+\frac{t \mu}{\lambda_{\alpha}}\right)^{n_{\alpha}}=\sum_{i_{\alpha}=0}^{n_{\alpha}}\left(\begin{array}{c}
n_{\alpha} \\
i_{\alpha}
\end{array}\right)\left(\frac{t \mu}{\lambda_{\alpha}}\right)^{i_{\alpha}}
$$

gives

$$
p_{b}=\frac{1}{\int_{0}^{\infty}\left(1+\frac{t \mu}{\lambda_{1}}\right)^{n_{1}} \ldots\left(1+\frac{t \mu}{\lambda_{k}}\right)^{n_{k}} \exp (-t) \mathrm{d} t} .
$$

\section{Arbitrary arrivals}

To utilize the calculations in Gopinath et al. [2], throughout this section the state of the switch is specified by the subset of idle links. For a state $I$ and link $i \in I(j \in I), I_{i}\left(I^{j}\right)$ denotes $I-i$ (resp. $\left.I \cup\{j\}\right)$. The instantaneous loss probability associated to link $i$ is $1-\epsilon_{i}$. A policy consists of specifying numbers $U\left(I, I_{i}\right)$ satisfying

$$
U\left(I, I_{i}\right) \geqslant 0, \sum_{i \in I} U\left(I, I_{i}\right)=1 \quad \text { if } I \neq \varnothing .
$$

Clearly this is no more general than the setup in section 2 . Let $R$ denote the matrix with entries

$$
R\left(I, I_{i}\right)=\epsilon_{i} U\left(I, I_{i}\right), R(\varnothing, \varnothing)=1
$$

and all other entries 0 .

\section{Deterministic arrivals}

If the switch is in state $I \neq \varnothing$ at time $t=0$ and calls arrive in a deterministic sequence at times $t_{1}<t_{2}<\ldots, t_{1} \geqslant 0$, we let $\tau_{U}(I)$ denote the time of the first visit to the blocked state $\varnothing$, when the policy is $U$. The numbers $p_{k}=\operatorname{Prob}\left(\tau_{U}(I)\right.$ $=t_{k}$ ) determine the distribution of $\tau_{U}(I)$. Let $M$ denote the matrix with entries

$$
\begin{aligned}
& M\left(J, J^{j}\right)=\mu \\
& M(J, J)=-(n-|J|) \mu
\end{aligned}
$$


and 0 elsewhere. Then we have

$$
\sum_{j=1}^{k} p_{j}=e_{I}^{T} \exp \left(M t_{1}\right) R \exp \left(M t_{2}\right) R \ldots \exp \left(M t_{k}\right) \operatorname{Re}_{\varnothing}
$$

where $e_{J}$ denotes the column vector with 1 in location $J$ and 0 elsewhere.

Following Gopinath et al. [2], for $d \geqslant 1$, let $N(J, d)$ denote the set of all $|J|$ tuples $\left\{n_{j}, j \in J\right\}$ such that $n_{j} \geqslant 1$ and $\sum_{j \in J} n_{j}=d$. Let $v_{d}$ denote the column vector with

$$
v_{d}(J)=(-1)^{|J|(d-1)} \sum_{N(j, d)} \prod_{j \in J} \epsilon_{j}^{n_{j}} .
$$

If we set $v_{0}=e_{\varnothing}$, then

$$
R v_{d}=v_{d+1}, 0 \leqslant d \leqslant n .
$$

(Equations (9) and (12) of Gopinath et al. [2]). Further, there are constants $c_{r}$, $r \geqslant 1$, (independent of the policy) such that

$$
M v_{d}=-\mu \sum_{r=1}^{d-1} c_{r} v_{d-r}-\mu(n-d) v_{d}, d \geqslant 0
$$

(Equation (14) of Gopinath et al. [2]). The independence of $p_{k}, k \geqslant 1$ of the policy can now be established from (3.1) by induction on $k$.

\section{Arbitrary arrivals}

If the switch is in state $I \neq \varnothing$ at $t=0$ and the input is an arbitrary arrival process independent of the holding times and routing decisions, it follows that the conditional distribution of the time to reach the blocked state, given the sequence of arrivals, is independent of the policy. Once in the blocked state, the switch remains blocked for an exponential holding time of mean $1 / n \mu$ independent of the arrival process, after which it enters one of the state $\{i\}, 1 \leqslant i \leqslant n$, with equal probability $1 / n$. Therefore, the distribution of the time of the second visit to the blocked state, conditioned on the sequence of arrivals, is independent of policy, and so on. Thus the law of overflow traffic is independent of policy.

\section{Renewal arrivals}

Suppose the arrival process is renewal. If the first call sees the switch blocked, the distribution of the state of the switch seen by the second call is independent of policy. Thus, if $N \in\{2,3, \ldots\}$ is the next blocked call, $E N$ is independent of policy. The blocking probability, $p_{b}=1 /(E N-1)$ is therefore independent of policy. It may be of some interest to calculate an explicit formula for it using the techniques of Takacs [5]. 


\section{An asymptotic formula for the blocking probability}

In this section we obtain an asymptotic formula for the integral in section 3 and thus for the blocking probability. Recall that there are $k$ trunks of $n_{1}, \ldots, n_{k}$ links respectively. To obtain an asymptotic formula we shall assume that $n_{i}=\alpha_{i}$ with $\alpha_{i} \geqslant 0, i=1, \ldots, k$ and $m$ goes to infinity. The integral in section 3 presents some problems, and can not be dealt with by means of a Laplace or saddle point expansion. This motivated us to prove the lemma below, for which we however later found the reference Sirovich [4]. In particular we obtain the following result form (2.5.10) of Sirovich [4] that (note that there is a misprint in (2.5.10), in view of $(2.5 .7))$ :

\section{LEMMA 1}

Let $R(x)=\int_{\alpha}^{\beta} \mathrm{e}^{h(x, t)} \mathrm{d} t$ where $h(x, t)$ is continuous in $x$ and twice continuously differentiable in $t$. Assume that for each fixed $x, h(x, t)$ has a single maximum point at $t_{0}=t_{0}(x)$ and that $0<\left|\partial^{2} h / \partial t^{2}\left(x, t_{0}(x)\right)\right|<\infty$. If

$$
\frac{\partial^{3} h}{\partial t^{3}}\left(x, t_{0}(x)\right)\left(t-t_{0}(x)\right)=O\left(\frac{\partial^{2} h}{\partial t^{2}}\left(x, t_{0}(x)\right)\right)
$$

as $x \rightarrow \infty$ for each fixed $t \in(\alpha, \beta)$ then

$$
R(x) \sim \mathrm{e}^{h\left(x, t_{0}(x)\right)} \int_{\alpha}^{\beta} \exp \left[\frac{\partial^{2} h}{\partial t^{2}}\left(x, t_{0}(x)\right) \frac{\left(t-t_{0}(x)\right)^{2}}{2}\right] \mathrm{d} t .
$$

We shall use the Vinogradov notation $f \ll g$ to mean that $f=O(g)$. With the notation as in section 3 let $p_{i}=\mu / \lambda_{i}, i=1, \ldots, k$. We use lemma 1 to prove the following asymptotic formula:

\section{THEOREM 1}

Assume $n_{i}=\alpha_{i} m, i=1, \ldots, k$ where $\alpha_{i}>0$ for all $i$ and $m \in \mathscr{N}$. Then for $p_{i}>0, i=1, \ldots, k$,

$$
\begin{aligned}
& \int_{0}^{\infty} \mathrm{e}^{-t}\left(1+t p_{1}\right)^{n_{1}} \ldots\left(1+t p_{k}\right)^{n_{k}} \mathrm{~d} t \sim \\
& \sqrt{2 \pi}\left(\prod_{j \leqslant k}\left(1+p_{j} w\right)^{n_{j}}\right) \mathrm{e}^{-w} /\left(\sum_{j \leqslant k} \frac{n_{j} p_{j}^{2}}{\left(1+p_{j} w\right)^{2}}\right)^{1 / 2}
\end{aligned}
$$

as $m \rightarrow \infty$ and where $w$ satisfies

$$
\sum_{j \leqslant k} \frac{n_{j} p_{j}}{1+p_{j} w}=1 .
$$


Proof

Define $h(x, t)$ by

$$
h(x, t)=x\left(\sum_{j \leqslant k} \alpha_{j} \log \left(1+t p_{j}\right)\right)-t
$$

for $x>0$ and $t>0$. Then,

$$
\begin{aligned}
& \frac{\partial h}{\partial t}=x \sum_{j \leqslant k} \frac{\alpha_{j} p_{j}}{1+t p_{j}}-1 \\
& \frac{\partial^{2} h}{\partial t^{2}}=-x \sum_{j \leqslant k} \frac{\alpha_{j} p_{j}^{2}}{\left(1+t p_{j}\right)^{2}} \\
& \frac{\partial^{3} h}{\partial t^{3}}=2 x \sum_{j \leqslant k} \frac{\alpha_{j} p_{j}^{3}}{\left(1+t p_{j}\right)^{3}} .
\end{aligned}
$$

From, (5.3) we see that the maximum $t_{0}=t_{0}(x)$ for $h(x, t)$ satisfies

$$
\sum_{j \leqslant k} \frac{\alpha_{j} p_{j}}{1+t_{0} p_{j}}=\frac{1}{x}
$$

which shows that as $x \rightarrow \infty, t_{0}(x) \rightarrow \infty$. Therefore, for $x$ sufficiently large,

$$
\frac{1}{x}=\sum_{j \leqslant k} \frac{\alpha_{j} p_{j}}{1+t_{0} p_{j}} \geqslant \frac{1}{2} \min _{j \leqslant k}\left(\alpha_{j} p_{j}\right)\left(\sum_{j \leqslant k} \frac{1}{p_{j}}\right) \frac{1}{t_{0}} .
$$

We also have trivially,

$$
\frac{1}{x}=\sum_{j \leqslant k} \frac{\alpha_{j} p_{j}}{1+t_{0} p_{j}} \leqslant \max _{j \leqslant k}\left(\alpha_{j} p_{j}\right)\left(\sum_{j \leqslant k} \frac{1}{p_{j}}\right) \frac{1}{t_{0}} .
$$

Therefore from (5.7) and (5.8) we have, solving for $t_{0}(x)$ and noting that $\alpha_{j}$ and $p_{j}$ are constants,

$$
\begin{aligned}
& t_{0}(x) \ll x \\
& t_{0}(x) \gg x .
\end{aligned}
$$

Hence, by (5.4) and (5.9), for $x$ sufficiently large,

$$
\left|\frac{\partial^{2} h}{\partial t^{2}}\left(x, t_{0}(x)\right)\right|=x \sum_{j \leqslant k} \frac{\alpha_{j} p_{j}^{2}}{\left(1+t_{0} p_{j}\right)^{2}} \gg \frac{1}{x} .
$$

Similarly by (5.5) and (5.10),

$$
\frac{\partial^{3} h}{\partial t^{3}}\left(x, t_{0}(x)\right)=2 x \sum_{j \leqslant k} \frac{\alpha_{j} p_{j}^{3}}{\left(1+t_{0} p_{j}\right)^{3}} \ll \frac{1}{x^{2}} .
$$


Thus by (5.9), (5.11) and (5.12) it follows that, for each fixed $t>0$,

$$
\frac{\partial^{3} h}{\partial t^{3}}\left(x, t_{0}(x)\right)\left(t-t_{0}(x)\right) \ll \frac{\partial^{2} h}{\partial t^{2}}\left(x, t_{0}(x)\right)
$$

and thus (5.1) of the theorem is satisfied. Thus, by lemma 1

$$
\begin{aligned}
\int_{0}^{\infty} \mathrm{e}^{h(x, t)} \mathrm{d} t & \sim \mathrm{e}^{h\left(x, t_{0}(x)\right)} \int_{0}^{\infty} \exp \left[\frac{\partial^{2} h}{\partial t^{2}}\left(x, t_{0}(x)\right) \frac{\left(t-t_{0}(x)\right)^{2}}{2}\right] \mathrm{d} t \\
& =c_{1}(x) \int_{0}^{\infty} \exp \left[-c_{2}(x) \frac{\left(t-t_{0}\right)^{2}}{2}\right] \mathrm{d} t
\end{aligned}
$$

where

$$
c_{1}(x)=\left(\prod_{j \leqslant k}\left(1+p_{j} t_{0}\right)^{\alpha_{j} x}\right) \mathrm{e}^{-t_{0}}
$$

and

$$
c_{2}(x)=\sum_{j \leqslant k} \frac{\alpha_{j} x p_{j}^{2}}{\left(1+t_{0} p_{j}\right)^{2}}
$$

by definition of $h(x, t)$ and (5.4). Now

$$
\begin{aligned}
& \int_{0}^{\infty} \exp \left[-\frac{c_{2}}{2}\left(t-t_{0}\right)^{2}\right] \mathrm{d} t \\
& \quad=\sqrt{\frac{2}{c_{2}}} \int_{-\sqrt{\frac{c_{2}}{2} t_{0}}}^{\infty} \exp \left(-u^{2}\right) \mathrm{d} u \\
& \quad=\sqrt{\frac{2}{c_{2}}}\left(\int_{-\infty}^{\infty} \exp \left(-u^{2}\right) \mathrm{d} u-\int_{\sqrt{\frac{c_{2}}{2}} t_{0}}^{\infty} \exp \left(-u^{2}\right) \mathrm{d} u\right) .
\end{aligned}
$$

Since, for $v \geqslant 1$,

$$
\int_{v}^{\infty} \exp \left(-w^{2}\right) \mathrm{d} w \leqslant \int_{v}^{\infty} w \exp \left(-w^{2}\right) \mathrm{d} w=\frac{1}{2} \exp \left(-v^{2}\right)
$$

it follows that,

$$
\int_{0}^{\infty} \exp \left[-\frac{1}{2} c_{2}\left(t-t_{0}\right)^{2}\right] \mathrm{d} t=\sqrt{\frac{2 \pi}{c_{2}}}+O\left(\exp \left(-\frac{1}{2} c_{2} t_{0}^{2}\right)\right)
$$

from (5.16). Therefore, by (5.13),

$$
\int_{0}^{\infty} \mathrm{e}^{h(x, t)} \mathrm{d} t \sim \sqrt{2 \pi} c_{1}(x) \frac{1}{\sqrt{c_{2}(x)}} .
$$


Thus, for $n_{i}=\alpha_{i} m, i=1, \ldots, k$,

$$
\begin{aligned}
& \int_{0}^{\infty} \mathrm{e}^{-t}\left(1+t p_{1}\right)^{n_{1}} \ldots\left(1+t p_{k}\right)^{n_{k}} \mathrm{~d} t=\int_{0}^{\infty} \mathrm{e}^{h(m, t)} \mathrm{d} t \\
& \quad \sim \sqrt{2 \pi} c_{1}(m) \frac{1}{\sqrt{c_{2}(m)}} \\
& \quad=\sqrt{2 \pi}\left(\prod_{j \leqslant k}\left(1+p_{j} w\right)^{n_{j}}\right) \mathrm{e}^{-w} /\left(\sum_{j \leqslant k} \frac{n_{j} p_{j}^{2}}{\left(1+w p_{j}\right)^{2}}\right)^{1 / 2}
\end{aligned}
$$

where $w$ satisfies (from 5.6)

$$
\sum_{j \leqslant k} \frac{n_{j} p_{j}}{1+w p_{j}}=1
$$

thus proving the theorem.

\section{References}

[1] V.E. Benes, Transition probabilities for telephone traffic, Bell System Technical Journal 38 (1959) 211-258.

[2] B. Gopinath, J.M. Garcia and P. Varaiya, Blocking probability in a switching center with arbitrary routing policy, AT\&T Bell Laboratories Technical Journal 63, No. 5 (May-June 1984) $709-720$.

[3] A.Y. Khintchine, Mathematical methods in the theory of Queueing (Griffin, London, 1960).

[4] L. Sirovich, Techniques of Asymptotic Analysis (Springer-Verlag, 1971).

[5] L. Takacs, Introduction to the Theory of Queues (Oxford University Press, New York, 1962). 